\title{
Plan para desarrollar redes de vinculación y gestión en Instituciones de I\&D en Salud: estudio de caso *
}

\author{
Plan to Develop Linkage and Project Management Networks in Health R\&D \\ Institutions: A Case Study
}

\author{
Luis Roberto Vega-González \\ Magíster en ingeniería de sistemas y magíster en administración de las organizaciones, \\ Instituto de Ciencias Aplicadas y Tecnología, \\ Ciudad de México, México, roberto.vega@icat.unam.mx
}

Roberto Misael Vega-Salinas

Magíster en administración, Instituto Nacional de Medicina Genómica, Ciudad de México, México, rvega@inmegen.gob.mx

\begin{abstract}
Cómo citar / How to cite
Vega-González, L. R.; Vega-Salinas, R. M.; (2020). Plan para desarrollar redes de vinculación y gestión en Instituciones de I\&D en Salud: estudio de caso. Revista CEA, v. 6, n. 12, 105-127. https://doi.org/10.22430/24223182.1504
\end{abstract}

Recibido: 6 de febrero de 2020

Aceptado: 28 de mayo de 2020

\section{Resumen}

En este artículo se presenta el caso del Instituto Nacional de Medicina Genómica (INMEGEN) de la Secretaría de Salud del Gobierno Federal Mexicano, en el cual se realiza Investigación y Desarrollo (I\&D) en Medicina Genómica. La vinculación del instituto ha sido tradicionalmente académica y de investigación. En el marco de tiempos de cambios internos y externos muy intensos, y considerando el esquema de austeridad económica planteada por el actual Gobierno, se requiere implementar acciones para mejorar su vinculación con el fin de propiciar la generación de nuevos proyectos y de ingresos externos en un esquema de gestión moderno. Para encontrar la propuesta de acciones que pudieran responder a esta necesidad, se propuso el desarrollo de un plan estratégico basado en el establecimiento de redes de innovación tecnológica. La metodología de planeación estratégica utilizada consistió en las siguientes etapas: (a) un diagnóstico para determinar la situación de referencia, (b) planteamiento de las condiciones para realizar la planeación, (c) la elaboración de un

\footnotetext{
* Este artículo se deriva del proyecto titulado «Proyecto de Desarrollo de Vinculación Horizontal del INMEGEN con Instituciones Nacionales e Internacionales» y ha sido financiado con recursos propios.
} 
plan normativo para llegar a la situación futura esperada y (d) el desarrollo de un plan táctico con acciones de corto, mediano y largo plazo. En conclusión, en el plan propuesto para incentivar, mejorar y sistematizar las acciones de gestión de proyectos y vinculación con la sociedad se encontró que se requiere de dos acciones fundamentales: (a) construir comunidades o redes de innovación con instituciones nacionales e internacionales públicas y privadas y (b) llevar a cabo un mayor acercamiento y contacto directo con el público usando unidades móviles y módulos itinerantes para llevar servicios de medicina genómica directamente a distintas zonas de la Ciudad de México y su área conurbada.

Palabras clave: planificación estratégica, redes de vinculación, salud.

Clasificación JEL: I19; M15.

\begin{abstract}
This paper presents the case of the National Institute of Genomic Medicine (abbreviated INMEGEN in Spanish) which is part of the Ministry of Health of the Federal Government of Mexico. This institute conducts Research and Development (R\&D) studies in genomic medicine. Its linkage activities have traditionally and primarily been focused on academic and research collaborations. However, given the intense internal and external changes and considering the economic austerity raised by the current government, further linkage activities should be implemented to promote new projects and generate external revenues under a modern administration scheme. To identify the actions that may help to address this need, we propose to develop a strategic plan based on technology innovation networks. The planning methodology includes four steps: (a) carrying out an analysis to determine the baseline situation, (b) establishing the conditions for planning, (c) designing a regulatory strategy to achieve the expected future situation, and (d) developing a tactical plan with short-, medium-, and long-term actions. In conclusion, the proposed plan to boost, improve, and systematize project management and linkage activities with society suggest the need to take two fundamental measures: (a) building innovation networks with national and international private and public institutions, and

(b) getting closer and having a direct contact with communities using mobile and itinerant units to bring genomic medicine services directly to various areas of Mexico City and its metropolitan area.
\end{abstract}

Keywords: strategic planning, linkage networks, health.

JEL classification: I19; M15.

\title{
1. INTRODUCCIÓN
}

El derecho a la salud es uno de los derechos principales para lograr que una sociedad goce de condiciones de vida mínimas y dignas que le permita el ejercicio real de sus libertades (Díaz Silva et al., 2012; MacNaughton \& McGill, 2019). Para hacer frente y atacar los problemas de salud pública, en la década de 1940, el gobierno mexicano sentó las bases del sistema de salud con la creación del Instituto Mexicano del Seguro Social, la conversión del entonces Departamento de Salubridad en la Secretaría de Salubridad y Asistencia y el surgimiento de los primeros Institutos Nacionales de Salud. En el año 2000, se expidió la primera Ley de los Institutos Nacionales de Salud. 
Estos institutos actualmente son regulados por la Ley de los Institutos Nacionales de Salud, publicada en el Diario Oficial de la Federación el 22 de junio de 2006 y son coordinados por la Secretaría de Salud. Su ámbito de cobertura es el territorio nacional y tienen como objetivo principal la investigación científica en el campo de la salud, la formación y capacitación de recursos humanos calificados y la prestación de servicios de atención médica de alta especialidad.

En 1943 se inauguró el Hospital Infantil de México Federico Gómez, considerado como el primer Instituto Nacional de Salud en México. Hoy en día el sistema de Institutos Nacionales de Salud está constituido por las siguientes once instituciones: el Instituto Nacional de Pediatría, el Instituto Nacional de Cardiología Ignacio Chávez, el Instituto Nacional de Psiquiatría Juan Ramón de la Fuente, el Instituto Nacional de Perinatología, el Instituto Nacional de Geriatría, el Instituto Nacional de Cancerología, el Instituto Nacional de Ciencias Médicas y Nutrición Salvado Zubirán, el Instituto Nacional de Enfermedades Respiratorias, el Instituto Nacional de Salud Pública, el Instituto Nacional de Neurología y Neurocirugía y el Instituto Nacional de Medicina Genómica (INMEGEN).

EI INMEGEN fue fundado en 2004 como resultado del trabajo previamente efectuado por el Consorcio Promotor del Instituto de Medicina Genómica, integrado por la Universidad Nacional Autónoma de México, el Consejo Nacional de Ciencia y Tecnología, la Secretaría de Salud, y la Fundación Mexicana para la Salud (Instituto Nacional de Medicina Genómica, s. f.). Para su creación, se llevó a cabo la evaluación de factibilidad, preparación y desarrollo de líneas de investigación en temas genómicos relacionados con cáncer pediátrico, gástrico, tumores de células, leucemias, arterioesclerosis y enfermedades metabólicas hereditarias (Jiménez, 2003; Taylor-Alexander \& Schwartz-Marín, 2013).

El instituto se creó con el objetivo de convertirse en el vehículo para el desarrollo de la medicina genómica, la investigación científica, la formación y capacitación de recursos humanos especializados, el desarrollo de tecnología y la vinculación con la industria para el desarrollo de productos y servicios de base genómica (Séguin et al., 2008). Su ámbito de acción comprende todo el territorio nacional. Las principales acciones que ha adelantado esta institución a lo largo de este tiempo han sido: (a) potencializar la medicina genómica o medicina personalizada como medicina de precisión al seleccionar tratamientos dirigidos especialmente para cada caso de acuerdo al concepto genético de su enfermedad; (b) desarrollar herramientas de diagnóstico y tratamiento genómico, como la farmacogenómica, entre otras, para ofrecer las bases para una práctica médica basada en la estructura genómica de las poblaciones mexicanas y en sus necesidades epidemiológicas; (c) establecer alianzas estratégicas para generar una vinculación horizontal en tres vertientes: académica, dependencias públicas y sectores productivos e industriales nacionales e internacionales relacionadas con la medicina genómica y áreas afines, las cuales han generado colaboraciones respaldadas por más de 360 instrumentos consensuales.

Hoy en día se considera que el INMEGEN es una de las principales instituciones en investigación genómica a nivel nacional e internacional, ya que ha contribuido a la generación de aplicaciones genómicas innovadoras para mejorar la atención de la salud. Por ejemplo, se tienen los trabajos sobre deficiencias en el DNA de Staines Boone et al., (2019); en poliformismos en infecciones humanas por Taenia solium realizados por Fleury et al., (2018); y de control del HIV en poblaciones genéticamente mezcladas de Valenzuela-Ponce, et al., (2018). También se cuenta con la solicitud de patente 
mexicana de un método de pronóstico de cáncer de mama (Meléndez Zajgla y Bahena Ocampo, 2015).

A finales de 2018, tomó posesión el licenciado Andrés Manuel López Obrador como presidente de México, encabezando lo que él llama la Cuarta Transformación de México (Villegas, 2018; Rojas, 2018). Este movimiento político-social se ha caracterizado por la austeridad y el combate contra la corrupción en todas las entidades y dependencias del sector público. Bajo esta racionalidad, se presentó el reto de generar un esquema de vinculación y gestión moderna para brindar la atención directa y personalizada a los usuarios, entre los que se encuentran los ciudadanos y las instituciones públicas y privadas que conforman los diferentes sectores productivos del país. Una de las finalidades es crear unidades de negocio y propiciar la generación de ingresos externos mediante la detección y desarrollo de nuevos proyectos, para dar continuidad las vinculaciones científicas y académicas existentes.

Con este objetivo, se hizo clara la necesidad de mejorar su sistema de vinculación y gestión de proyectos tecnológicos. Cuando las instituciones de I\&D de alto nivel son jóvenes o de reciente creación, el primer obstáculo para su vinculación es que, para este tipo de instituciones, no hay «clientes» si se toca directamente a la puerta y se solicita el desarrollo de nuevos proyectos de I\&D. ¿Qué hacer? ¿Cómo atraer clientes? ¿Cómo establecer alianzas estratégicas para cumplir con la misión y los objetivos organizacionales? ¿Cómo promover la vinculación de la institución para lograr la concertación y realización de nuevos proyectos? ¿Cómo acercarse a la población y desarrollar nuevas modalidades de atención y servicios? ¿Cómo desarrollar unidades estratégicas de negocios para obtener ingresos extraordinarios que permitan mejorar los servicios institucionales en un circuito virtuoso? En este contexto, el objetivo de este trabajo fue desarrollar un plan estratégico para mejorar y potenciar la vinculación de la organización usando un esquema de redes abiertas y acciones puntuales para permitir un mayor acercamiento a la sociedad.

\section{MARCO TEÓRICO}

\section{Planeación estratégica}

En el último cuarto del siglo pasado se dio un fenómeno de cambios intensivos en los ámbitos tecnológico, económico y organizacional a nivel mundial. Para Aguilar Cruz (2000), la necesidad de planear, esencialmente, deriva del hecho de que toda organización opera en un medio que experimenta constantes cambios de tipo tecnológico y político, en el grado y carácter de la competencia, en las actitudes o normas sociales y en la actividad económica, resultado del fenómeno de globalización.

Por otra parte, para Hofer y Schendel (1985), el concepto de estrategia está estrechamente ligado al concepto de supervivencia, es decir: "a la larga solo sobreviven aquellas organizaciones que sirven a las necesidades de la sociedad efectiva y eficientemente, esto es, suministran los beneficios que la sociedad exige (...)».

Para Mintzberg (1994), es fundamental determinar el concepto de estrategia como el proceso de puesta en acción a partir de un planteamiento racional, en el que también se establezcan las prioridades tecnológicas. Según él, la planeación estratégica debería llamarse programación 
estratégica, ya que es un medio de programar las consecuencias de las estrategias creadas notablemente a través de la visión del líder que tomará las acciones.

Para Morcillo Ortega (1997), la dirección estratégica tiene que ver con la definición de estrategias competitivas para desarrollar nuevos productos o servicios y explotarlos rápidamente, dando origen a nuevas competencias de origen tecnológico en la organización. Para el autor, en un mundo dinámico con cambios tecnológicos intensos, la supervivencia debe ser parte de la misión de la organización y coincide con que la innovación es factor de la supervivencia. Al respecto, Sanders (1998) señala: «problemas y crisis parecen salir de todas partes (...) y la competencia es fiera». En lugar de esperar milagros, lo que se necesita es una forma de ver, entender y responder al gran medioambiente en tiempo real y pensando en el futuro, a través de un nuevo paradigma de planeación que ayude a la organización a identificar, responder y provocar cambios en su medio ambiente externo.

Stern y Stalk (1998) mencionan que, para Bruce Henderson, fundador de la firma Boston Consulting Group (BCG), la respuesta a la pregunta «¿qué es estrategia?» es: «los competidores que persisten en el tiempo, tienen que mantener, por diferenciación, una ventaja singular sobre todos los demás, la esencia de la estrategia es el manejo de la diferenciación». La estrategia organizacional radica en la forma de enfrentar a la competencia. Para esta firma, los elementos fundamentales de la competencia estratégica son: (1) La capacidad de entender la interacción entre competidores como un sistema dinámico completo en los que interactúan clientes, dinero, personas y recursos; (2) la capacidad de hacer uso de este conocimiento para predecir las consecuencias de una intervención concreta en el sistema y las nuevas formas de equilibrio dinámico estable que resultarán de esa intervención; (3) la disponibilidad de recursos no comprometidos que pueden dedicarse actualmente a diferentes usos y fines, aun en el caso de que su dedicación sea permanente y se retrase la obtención de beneficios; (4) la capacidad de predecir el riesgo y el rendimiento con suficiente exactitud y confianza como para justificar el uso de dichos recursos y (5) la buena disposición de actuar decididamente y comprometer dichos recursos.

El concepto de planeación estratégica surge de la necesidad de dirigir organizaciones complejas en un ambiente altamente dinámico. De manera que, para que una organización compleja como un instituto de I\&D en el área de la salud pueda sobrevivir, se requiere realizar planeación estratégica formal con los siguientes beneficios: (a) ayuda a la formulación de metas y objetivos organizacionales; (b) ayuda a identificar los grandes problemas; (c) ayuda a asignar los recursos estratégicos direccionalmente; (d) facilita la integración de diversas actividades administrativas y operativas. Así, cuando en una organización se identifica un problema, a través de la planeación estratégica se formulan e implantan acciones estratégicas para su solución.

En la práctica, la puesta en operación de los resultados de la planeación estratégica debe ser realizada por los usuarios interesados, quienes tienen necesidades determinadas y específicas que resolver, identificadas en el proceso de planeación.

\section{Metodologías de planeación estratégica}

Además de la metodología de competencia estratégica del BCG, existe una gran diversidad de metodologías para implementar el ahora llamado proceso de planeación estratégica. Algunas 
metodologías proponen procedimientos holísticos y comprensivos, como en el caso de la propuesta por Chiavenato (2017), quien hace una revisión de las escuelas de planeación estratégica, de la cual concluyen que los elementos del proceso son: (a) definir la misión organizacional, (b) definir la visión organizacional, (c) realizar el diagnóstico externo, (d) realizar diagnóstico interno, (e) identificar los determinantes del éxito, (f) definir los objetivos, (g) formular las estrategias, (h) formalizar el plan estratégico y (i) auditar el desempeño y resultados. Otras metodologías son más focales y sucintas, como la propuesta por Sabater Quinto y Monserrat Gauchi (2017) para realizar la planeación estratégica de la comunicación en redes sociales, quienes solo proponen un proceso consistente de los siguientes elementos: (a) análisis de la situación de la organización y sus productos, (b) diagnóstico, (c) determinación de objetivos y necesidades, (e) estrategias que seguir e implementación del plan, (f) revisión y evaluación del plan. La metodología propuesta por Kenny (2005) es intermedia, es decir, no es tan amplia ni tan reducida; consta de cinco procesos: (a) diagnóstico, (b) condiciones para realizar la planeación, (c) plan normativo, (d) plan táctico y (e) evaluación.

Por otra parte, cabe mencionar que, en la práctica, dependiendo del caso, cada uno de los subprocesos de la planeación estratégica se pueden desarrollar usando distintas herramientas, por ejemplo, para la definición de los horizontes de planeación se puede utilizar el método de escenarios, para el desarrollo de estrategias se puede usar una matriz de opciones y un árbol de decisiones probabilístico. También se puede hacer uso de modelos y algoritmos financieros, matrices de participación, análisis de mercado y competencia, de rentabilidad y tráfico, entre muchos otros. Sin embargo, el análisis de fortalezas, debilidades, oportunidades y amenazas (FODA), es una técnica de uso común que se utiliza durante el proceso de diagnóstico (Taylor, 1991).

\section{Comunidades de innovación y alianzas estratégicas}

Con el advenimiento del Internet a fines del siglo pasado, se dispuso de una tecnología de comunicaciones sumamente poderosa que permitía la comunicación instantánea entre individuos y organizaciones a cualquier distancia y en cualquier parte del mundo. El mundo entero se convirtió en una aldea global y esto influyó naturalmente en las técnicas de vinculación y gestión de proyectos.

Hoy en día, los proyectos de I\&D para la Innovación no solo se dan en las Instituciones de Educación Superior, sino que han permitido que las Comunidades de Innovación abierta se hayan ampliado hacia otros miembros y sectores de la sociedad. Domínguez Ríos (2013), menciona que el concepto de innovación abierta, es el uso de los flujos de conocimiento que ingresan y salen de la organización con el propósito de acelerar internamente la innovación y expandir los mercados que puedan hacer uso externo de la innovación. En otras palabras, la innovación abierta permite a las organizaciones hacer uso de recursos de conocimiento de otros usuarios para crecer y prosperar en el largo plazo. (Wu \& Gong, 2019; Giordani et al., 2018).

En ese sentido, Campos Ríos y Sánchez Daza (2005) consideran que si bien es cierto que la vinculación es una función sustantiva de las Instituciones de Educación Superior, esto las obliga a formar 'redes de acción' que tienen influencia más allá de la propia universidad; a saber, deben desarrollar programas para relacionarse intensamente con otros agentes como el Gobierno, los sectores productivos, el sistema educativo en su conjunto $y$, sobre todo, los centros e instituciones de 
investigación del nivel superior e incluso sectores de la sociedad que puedan colaborar en una estructura realmente operativa.

Lynn et al., (1996) han discutido la importancia del medio ambiente institucional para la comercialización o la transferencia de los resultados de proyectos de innovación, a través de lo que llaman la «comunidad de innovación». El desarrollo de estas comunidades ayuda a la rápida introducción y difusión de las tecnologías en la sociedad. Construir una comunidad de innovación permite generar e introducir nuevas variables como la expansión de las capacidades tecnológicas de la organización y contar con tecnologías de producción adicionales y nuevas estrategias de innovación y desarrollo. Por supuesto, hay que tener en cuenta que el sistema de innovación no es estático y es menester resolver el problema de las relaciones interorganizacionales para su buen funcionamiento. Los autores puntualizan que se necesitan varios actores para contar con la cantidad de componentes y servicios que se requieren para crear una nueva tecnología. Estos actores organizacionales constituyen la llamada «comunidad de innovación».

Las comunidades de innovación son financiadas por los Ministerios y entidades garantes de Ciencia y Tecnología en prácticamente todos los países de Latinoamérica. Por ejemplo, Rodríguez et al., (2015) indican que en Nicaragua las administraciones públicas como el Ministerio de Fomento, Industria y Comercio de la Dirección General de Industria y Tecnología han potenciado la cooperación entre universidades, organismos públicos de investigación y empresas mediante instrumentos específicos de financiación de proyectos, redes y alianzas estratégicas.

Pero no todo es tan sencillo, es importante tener en cuenta que cuando las organizaciones se involucran en diferentes acciones de cooperación de Investigación y desarrollo (I\&D), con diferentes socios o contrapartes; esto puede tener algunos efectos benéficos y algunos otros en detrimento del desempeño organizacional. Por ejemplo, Kaats y Opheij (2012) mencionan que cuando dos organizaciones que colaboran son diferentes en cuanto a su tamaño, objetivos, recursos humanos y estructura, se pueden crear problemas de ambigüedad; por lo tanto, es necesario usar un lenguaje que propicie un clima organizacional que facilite la colaboración, debido a que la diferencia de lenguajes, expectativas y objetivos fomenta la confusión.

Belderbos et al., (2006) proponen que es muy importante tener una estrategia específica y racional de alianzas estratégicas, ya que, tratándose de empresas, la cooperación con clientes de tamaño medio persigue la aceptación en el mercado y la difusión de productos innovadores, mientras que cooperar con firmas pequeñas puede tener altos costos y las complejidades de manejar muchas sociedades con diversos objetivos de innovación. Estos autores proponen que es muy importante que las alianzas establecidas para realizar acciones de I\&D sean complementarias.

Entonces, para llegar a la complementariedad, la cooperación entre institutos de investigación resulta más natural con universidades que con empresas o firmas innovadoras y generalmente busca innovaciones de producto tipo ruptura que puedan abrir nuevos mercados o segmentos de mercado. También pueden ser socios de empresas en campos tecnológicos nuevos. Belderbos et al., (2006) citan a Leyden y Link (1999), quienes estudiaron el caso de un Instituto de Investigación Público asociado con múltiples socios en un Proyecto de Investigación Conjunta o Research Joint Venture (RJV), encontrando que el incremento en el número de firmas asociadas implica, asimismo, un incremento significativo de los costos de monitoreo y problemas de apropiación de la propiedad 
intelectual de los resultados de la investigación. Además, el manejo de sociedades de I\&D con múltiples socios deriva en un aumento en los costos de complejidad y de coordinación.

Por otra parte, Yusuf (2008) subraya la importancia de los procesos de intermediación en el intercambio de conocimiento que se da entre universidades y negocios como un medio mediante el cual se desarrolla nuevo conocimiento que puede llevar a innovaciones comerciales. Yusuf menciona que los estudiantes son uno de los mejores medios para la transferencia de los resultados de investigación y el valioso conocimiento tácito asociado. También son un canal para la transmisión de conocimiento de las firmas hacia la universidad o, por extensión, hacia los institutos de investigación, canal que requerirá de muchas investigaciones futuras. Esta postura es claramente asumida por Velázquez (2012), quien enfatiza fuertemente en la importancia de la comunicación en el proceso de vinculación y establece que la comunicación debe ser abierta, evolutiva, flexible, multidireccional e instrumentada.

Kaats y Opheij (2012) definen la colaboración entre las organizaciones como una forma de organización en la que el personal de organizaciones autónomas llega a acuerdos duraderos y, al hacerlo, armonizan mutuamente elementos del trabajo entre ellos. Esto da como resultado una amplia gama de asociaciones de colaboración con una intención duradera, pero con duración limitada. Kaats y Opheij (2014) también definen cinco características de colaboración: (1) un alto grado de interdependencia, (2) un oscuro centro de poder, (3) una nueva realidad, (4) el poder atractivo de la heterogeneidad y (5) un contexto en flujo constante.

Hace algunas décadas, la fuente de innovación para las organizaciones era únicamente sus capacidades internas de I\&D, por lo que se producía una interacción limitada con los actores externos. Chang et al., (2002) presentaron un programa de asociación público-privada para el desarrollo de tecnología en etapa temprana. En la actualidad, las organizaciones públicas y privadas están yendo más allá de sus límites organizativos y se están involucrando en colaboraciones interorganizacionales para obtener conocimiento e innovaciones. Esto se conoce como innovación abierta (Chesbrough et al., 2006; Chesbrough \& Appleyard, 2007; Lichtenthaler, 2017). Las alianzas estratégicas bien pueden haberse convertido en una de las estrategias empresariales más comúnmente adoptadas durante la primera década del 2000, produciendo una proliferación de actividad colaborativa. Por ejemplo, cada año se crean más de 10000 alianzas nuevas en las áreas de biotecnología y Arthur D. Little y Price Waterhouse Coopers estiman que entre el 25 y el $50 \%$ del presupuesto de I + D farmacéutica se gasta en alianzas (De Rond y Bouchikhi, 2004).

Recientemente se ha investigado mucho sobre diversos temas en torno a las alianzas tecnológicas para organizaciones privadas y públicas y su gobernanza (Farazi et al., 2019). Los temas de investigación han incluido información acerca de cómo se inician, operan, evalúan las alianzas, así como los diversos factores internos y externos que influyen en el progreso del desarrollo de la alianza y las bases para un modelo de evolución estratégica de la alianza (Wohlstetter et al., 2005).

Kim (2015) desarrolló una interesante área de investigación sobre el desarrollo de un modelo de financiamiento del desarrollo de tecnología de equipamiento médico (MET) y sus transferencias a países en desarrollo en una asociación público-privada. Dado que las redes colaborativas se volvieron cada vez más grandes, Calvin et al., (2014) abordaron el tema de la importancia del papel que lleva la empresa dentro de la red de alianzas, central o periférica, y Kim y Choi (2014) han propuesto 
configurar una cartera de las alianzas tecnológicas que tiene la organización. Lerner y Merges (1998) han señalado la necesidad de asignar cuidadosamente los acuerdos contractuales para las alianzas tecnológicas y el derecho a tomar decisiones sobre cuestiones que no pueden especificarse contractualmente o los derechos de control.

Das y Kumar (2011) señalan que es necesario reconocer que los socios de la alianza se encuentran en un estado de interdependencia que puede ser un precursor de posibles conflictos; por lo tanto, otro tema crítico es cómo se debe manejar la interdependencia que existe entre los socios. Los autores concluyen que la gestión de la interdependencia requiere habilidades extraordinarias de negociación. Aunque la definición de colaboración dada por Huxham y Vangen (2005) es simple, "cualquier situación en la que las personas trabajen a través de las fronteras organizacionales hacia un fin positivo», comenzar y mantener una colaboración no lo es. En primer lugar, se requiere definir la necesidad y las características y razones esenciales para colaborar a fin de minimizar los malentendidos y la discordia resultante que a menudo rodea las relaciones interorganizacionales. En esencia, la colaboración consiste en establecer conexiones entre personas, entre comunidades, y con el mundo actual. En ese sentido, las alianzas estratégicas son colaboraciones voluntarias entre organizaciones que implican intercambio de productos, intercambio o desarrollo conjunto, desarrollo de tecnología o la prestación de servicios que persiguen un conjunto de objetivos común. Para Lin y Darnall (2014), las motivaciones para formar alianzas estratégicas son: la participación de los riesgos de toma de decisiones, basados en los recursos institucionales disponibles, los deseos de mejorar las competencias internas y el aprendizaje organizacional.

Cada día, más organizaciones de salud pública participan en la construcción de alianzas y redes para la innovación y la producción de conocimiento (Suárez-Balcázar et al., 2005); las alianzas innovadoras, la investigación participativa y las alianzas tecnológicas para la salud pública han sido ampliamente discutidas desde el último cuarto del siglo XX. Nelson et al., (1999) hablan sobre la posición de las agencias de salud pública estadounidenses cuando se establecen asociaciones estratégicas, concluyendo que antes de implementar cambios en el funcionamiento, la estructura o estrategias futuras, las agencias de salud deben evaluar su preparación interna para comprometerse a crear estas alianzas, que significan cambios organizacionales sustanciales.

\section{Las redes de innovación, fundamento de la innovación abierta}

El concepto de innovación abierta se desarrolló inicialmente para firmas manufactureras e industriales. Chesbrough et al., (2006) mencionan que el concepto de Open Innovation es el uso de los flujos de conocimiento que ingresan y salen de la empresa con el propósito de acelerar internamente la innovación y expandir los mercados que puedan hacer uso externo de la innovación. Es un paradigma que asume que las empresas podrían y deberían usar las ideas externas, así como también las internas, además de las rutas de mercado internas y externas, de tal forma que busquen avanzar tecnológicamente.

Con base en lo anterior, se podría extender este enfoque a lo que sucede en el ámbito de la ciencia, ya que también parece que ninguna institución que desarrolle ciencia de frontera puede desarrollar y obtener resultados científicos de alto impacto por sí misma, y requiere de trabajar en redes para obtener tal efecto. Por esa razón, actualmente, uno de los nuevos indicadores que permiten evaluar el desempeño de los institutos de I\&D es el tipo y el número de redes que tiene establecidas, los 
objetivos de las mismas, así como de sus resultados y tiempo de supervivencia. También es importante estudiar la forma de operación y de dirimir conflictos en las redes, pues la coordinación efectiva y la combinación de diferentes competencias es el elemento central para adelantar una innovación abierta exitosa.

Por otra parte, Petraite (2013) señala que hoy en día los sectores de alta tecnología se caracterizan por que los avances científicos y tecnológicos se dan muy rápidamente y el conocimiento básico requerido para su desarrollo está distribuido ampliamente, por lo que ninguna firma o institución posee toda la información relevante para llevar a cabo innovaciones exitosas internamente. En este contexto, aparece el concepto de innovación abierta, que se basa en el desarrollo de redes y en la aportación de la capacidad de producción o manufactura para desarrollar Joint Ventures de alta tecnología, en los cuales se pueda desarrollar conocimiento compartido y confiable adelantando procesos de investigación y desarrollo rápidos, además de acceso al mercado y a fuentes de comercialización. En las redes las nuevas ideas se desarrollan, se prueban y se implementan.

Sin embargo, Petraite (2013) indica que la transferencia de conocimiento de I\&D que se da en comunidades sociales no se da automáticamente, ya que requiere del desarrollo de importantes condiciones previas, como son la capacidad de absorber e integrar varias fuentes de conocimiento para la innovación, además de ajustar el comportamiento de las organizaciones en las redes colaborativas, de tal manera que acepten e incluyan el alineamiento y desarrollo de sus estructuras organizacionales y culturales para lograr la colaboración y la transferencia del conocimiento.

Vanhaverbeke et al., (2008) y Van de Vrande et al., (2006), citados por Petraite (2013), mencionan que depende de las necesidades y requisitos de desarrollo de conocimiento de la organización innovadora la forma de relacionarse con universidades, instituciones de investigación y las relaciones diádicas con otras firmas, creando redes o adquiriendo empresas start-ups tecnológicas. Para estos autores, desde el punto de vista de los recursos, hay tres razones de importancia para establecer enlaces interorganizacionales. Primero, en las redes se pueden generar equipos con otras empresas para monitorear y estar en contacto con los últimos desarrollos tecnológicos. Segundo, la colaboración permite la transferencia y absorción de conocimiento externo y actúa como un importante suplemento para las capacidades innovativas internas de las organizaciones. Tercero, las empresas ya no son capaces de explotar y comercializar productos innovativos por su cuenta.

\section{METODOLOGÍA}

Para diseñar el plan para implantar un nuevo sistema o modelo de vinculación y gestión en el instituto, se propone la metodología mostrada en la Tabla 1, que derivó de la secuencia de pasos de planeación estratégica propuesta por Kenny (2005). En esta, el diagnóstico del primer punto incluye la identificación de interesados clave y la identificación de factores estratégicos para estos interesados. En el punto número 2 de la metodología se establecen las condiciones para realizar la planeación, se incluye la calificación de desempeño de estos factores y la identificación de objetivos. En el plan normativo del punto número 3 se desarrollan las estrategias y se ajustan de acuerdo con los objetivos. El plan táctico del punto número 4 corresponde con el plan de acción. 
Tabla 1. Metodología de planeación utilizada

Table 1. Planning methodology implemented

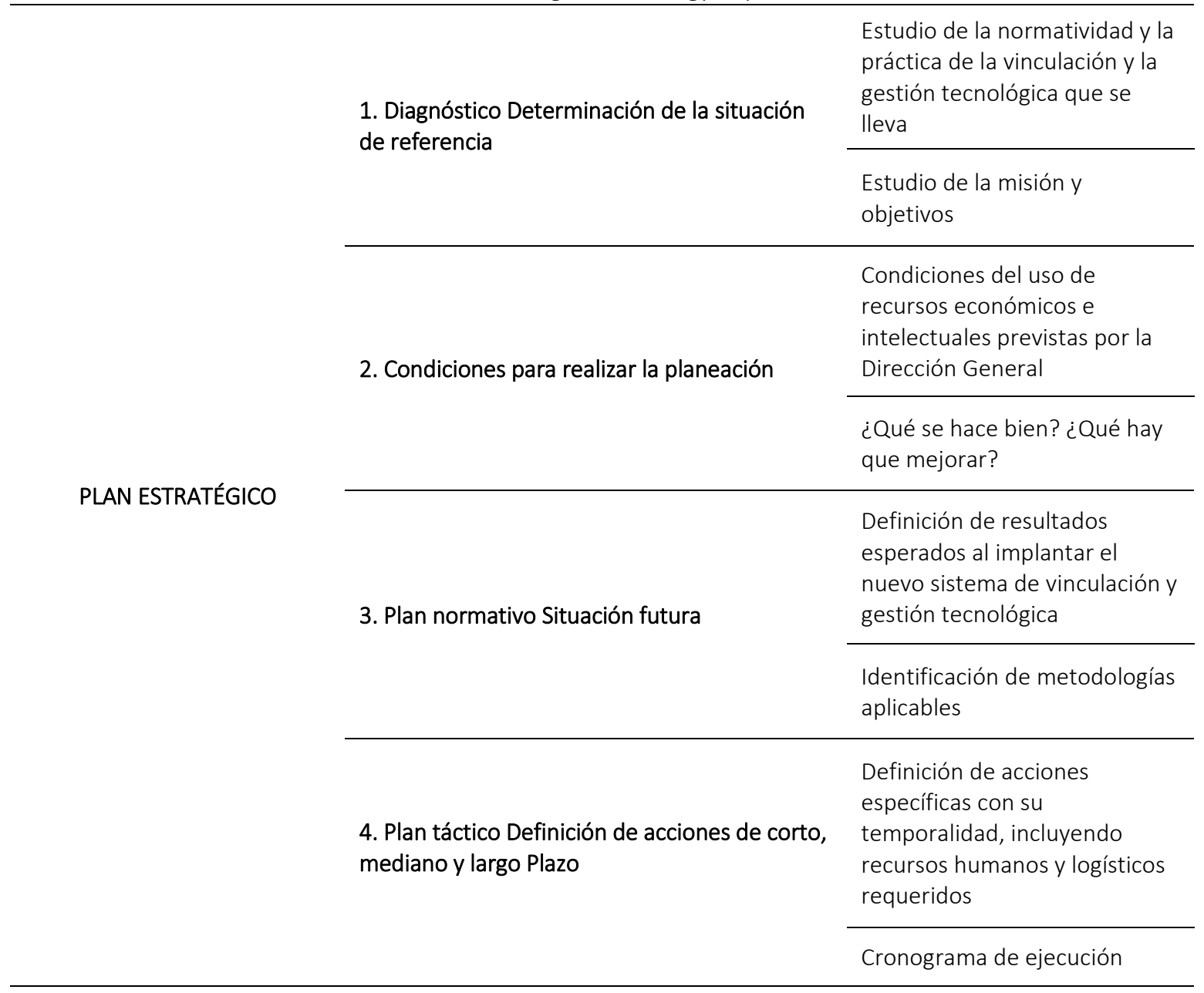

Fuente: elaboración propia.

El primer punto de la planeación estratégica es determinar con la mayor claridad posible cuál es la situación de referencia; es decir, hay que hacer un diagnóstico de la normatividad y la práctica mediante la cual se hace la vinculación y la gestión tecnológica en la institución. Actualmente, se está llevando a cabo un ejercicio de consenso con la participación de los directivos de la institución, y se propone la realización de encuestas a una muestra de académicos del instituto y responsables de instituciones, con las cuales el instituto tiene convenios de colaboración, y una encuesta simple a una muestra de cien personas del público general para tener una primera idea de qué tan conocido es el instituto y sus servicios. A partir de este diagnóstico, se debe determinar cuáles son las fortalezas y las debilidades del sistema actual.

Debido a las restricciones económicas y para la contratación de nuevo personal del programa de austeridad del nuevo Gobierno, el segundo punto sería tener en cuenta claramente las condiciones establecidas por la dirección para llevar a cabo la planeación del nuevo sistema de vinculación y gestión tecnológica; asimismo, responder a las preguntas: ¿Qué se hace bien? ¿Qué hay que mejorar? 
La tercera etapa de la planeación se basaría en plantear la situación futura esperada tanto en la definición del objetivo como de los resultados esperados con la implantación del nuevo sistema de vinculación y gestión tecnológica, con base en los plazos de ejecución e identificando las posibles oportunidades y amenazas.

Finalmente, se debe llevar acabo la definición de las distintas acciones de corto, mediano y largo plazo que se deberían realizar, incluyendo la planeación del personal participante, el presupuesto de gasto corriente y de inversión requerido, así como la logística y las responsabilidades.

\section{RESULTADOS}

En las siguientes secciones se presentan de forma resumida los resultados obtenidos en la elaboración del plan.

\section{Diagnóstico}

La vinculación en el Instituto se ha dado en diversas formas desde su creación. Una de sus áreas de vinculación principales ha sido la firma de convenios de colaboración de I\&D con otros Institutos Nacionales de Salud (INS) en México. A continuación, se presentan algunos ejemplos de Convenios Marcos vigentes entre el INMEGEN y los INS que buscan impulsar la vinculación y desarrollar proyectos mediante redes entre los mismos investigadores: (1) Instituto Nacional de Cancerología (2010), (2) Instituto Nacional de Ciencias Médicas y Nutrición Salvador Zubirán (2010), (3) Instituto Nacional de Enfermedades Respiratorias (2010), (4) Instituto Nacional de Pediatría (2011)

El objeto de los instrumentos mencionados versa en materia de: (a) investigación científica, (b) intercambio académico/estancias, (c) desarrollo científico y tecnológico/proyectos, (e) enseñanza y divulgación. Hasta la fecha, el INMEGEN ha consolidado más de 360 convenios de colaboración con diversas entidades nacionales e internacionales buscando fortalecer sus lazos con relaciones de buena fe y largo aliento.

Es importante mencionar que el Instituto ha formado parte de importantes proyectos de colaboración en materia de genómica nacionales e internacionales, como el Consorcio Internacional del Genoma del Cáncer para la Medicina, la Alianza Global para Genómica y Salud y el Proyecto Genoma Humano. No obstante, en el Instituto se busca seguir enriqueciendo las relaciones de vinculación mediante colaboraciones entre los mismos investigadores, Instituciones de Educación Superior, Centros de Investigación, Asociaciones Civiles y Sectores Productivos con el objetivo de seguir generando productos y servicios de base genómica que sean para el beneficio de la salud de la sociedad. Para identificar las fortalezas y oportunidades en materia de vinculación y gestión tecnológica, se hizo referencia al Estatuto Orgánico del Instituto Nacional de Medicina Genómica publicado en marzo de 2011 (INMEGEN, 2011).

En el artículo 32 del Estatuto Orgánico se indica que:

\footnotetext{
${ }^{1}$ Para mayor información en torno a los resultados de vinculación que se han concretado en el INMEGEN por medio de convenios formalizados vigentes con otras entidades, favor de dirigirse al Sistema de Portales de Obligaciones de Transparencia del Gobierno de México (INAI), por medio del siguiente link: https://consultapublicamx.inai.org.mx/vut-web/ 
...la Dirección de Vinculación y Desarrollo Institucional (DVDI) podrá proponer y coordinar la vinculación horizontal del Instituto con los sectores público, social y privado, tanto nacionales como extranjeros, así como formalizar los convenios de colaboración necesarios que favorezcan el desarrollo de la investigación científica, nuevos negocios de base genómica y la formación de recursos humanos de alta especialidad en medicina genómica y coordinar y establecer la vinculación en acciones de asesoría y consulta en materia genómica entre el Instituto y los sectores público, social y privado del país... (p. 27).

Estas funciones son tan solo algunas de las que le corresponden a la DVDI; en cuanto a la parte administrativa de esta dirección, se refieren a acciones de planeación, dirección, representación jurídica, en comités directivos y de evaluación de propiedad intelectual. Se vislumbra un área de oportunidad para la función de vinculación en la institución.

\section{Condiciones para llevar a cabo el plan}

Las condiciones para desarrollar el plan fueron las siguientes: (a) debido a la austeridad impuesta por el nuevo gobierno a todas las instituciones del sector público, incluyendo al sector de la salud, la implementación del plan no debería ser costosa, (b) en su mayor parte las acciones deberían ser llevadas a cabo por el personal con el que ya cuenta la institución, (c) utilizar como referentes los modelos de gestión tecnológica de frontera, adaptados a las condiciones de la institución, (d) a través del modelo de gestión, desarrollar la difusión, y un mejor contacto con la población para instituciones de I\&D de alta tecnología en el sector salud, con las cuales ya tiene convenios firmados el instituto.

\section{Situación futura esperada}

A partir de reuniones entre los directivos y los miembros de la Dirección de Vinculación, aplicando metodología Delphy, se dio respuesta a las siguientes preguntas:

¿Dónde queremos estar en el corto plazo?

En un año se debe tener la especificación básica de la Red Tecnológica y del sistema de cómputo. Se debe haber entrevistado las diferentes áreas de vinculación de las instituciones de salud, universidades, empresas con las que el instituto ya colabora para invitarlos a formar parte de la Red. Se espera que en una primera fase se integren entre quince y treinta nodos. Se realizarán pruebas de comunicaciones y difusión de noticias de la red referentes a proyectos de I\&D y servicios disponibles que ofrece el instituto.

Para el segundo año se habrán diseñado, construido o adquirido los medios de la red para hacer promoción directa al público. En el segundo año se deberá presupuestar y construir la unidad móvil piloto. A partir del tercer año y hasta el quinto, se deberá diseñar un plan de visitas de la unidad a los distintos miembros de las redes nacionales.

\section{Plan normativo}

La estrategia central del plan propuesto fue el establecimiento de redes tecnológicas de innovación, siguiendo los modelos actuales de innovación abierta. La tecnología requerida para llevar a cabo estas 
redes descansará en un sistema de cómputo (software-hardware) para la administración, manejo y comunicaciones con todos los miembros de las redes, según su jerarquía; es decir, redes académicas o científico-tecnológicas con instituciones públicas o privadas nacionales o internacionales o redes persona a persona.

En el sistema se dispondrá de bases de datos de servicios, como la consultoría o los que brindan los laboratorios disponibles en el instituto. Se podrían incluir las noticias y las acciones de formación de recursos humanos. Se propone el uso intensivo de videoconferencias para la formación de recursos humanos. Este sistema de cómputo puede desarrollarse por personal del mismo instituto o bien con el apoyo de otra institución dedicada al desarrollo de sistemas computacionales, y gestionando recursos de los fondos de innovación federales o de la ciudad de México.

En la Figura 1, se observa la idea propuesta: es que, a través de la Dirección de Vinculación y Desarrollo Interinstitucional del Instituto, se coordine y gestione el establecimiento de las Redes de I\&D; asimismo, usar un modelo probado de gestión de proyectos para mejorar la entrega de resultados a la sociedad. La Dirección de Vinculación coordinará, en conjunto con las autoridades administrativas y bajo la supervisión de la Dirección de Investigación del Instituto, la comunicación con los académicos o grupos de académicos que actuarán como nodos de contacto de las diferentes redes que existan o sean conformadas.

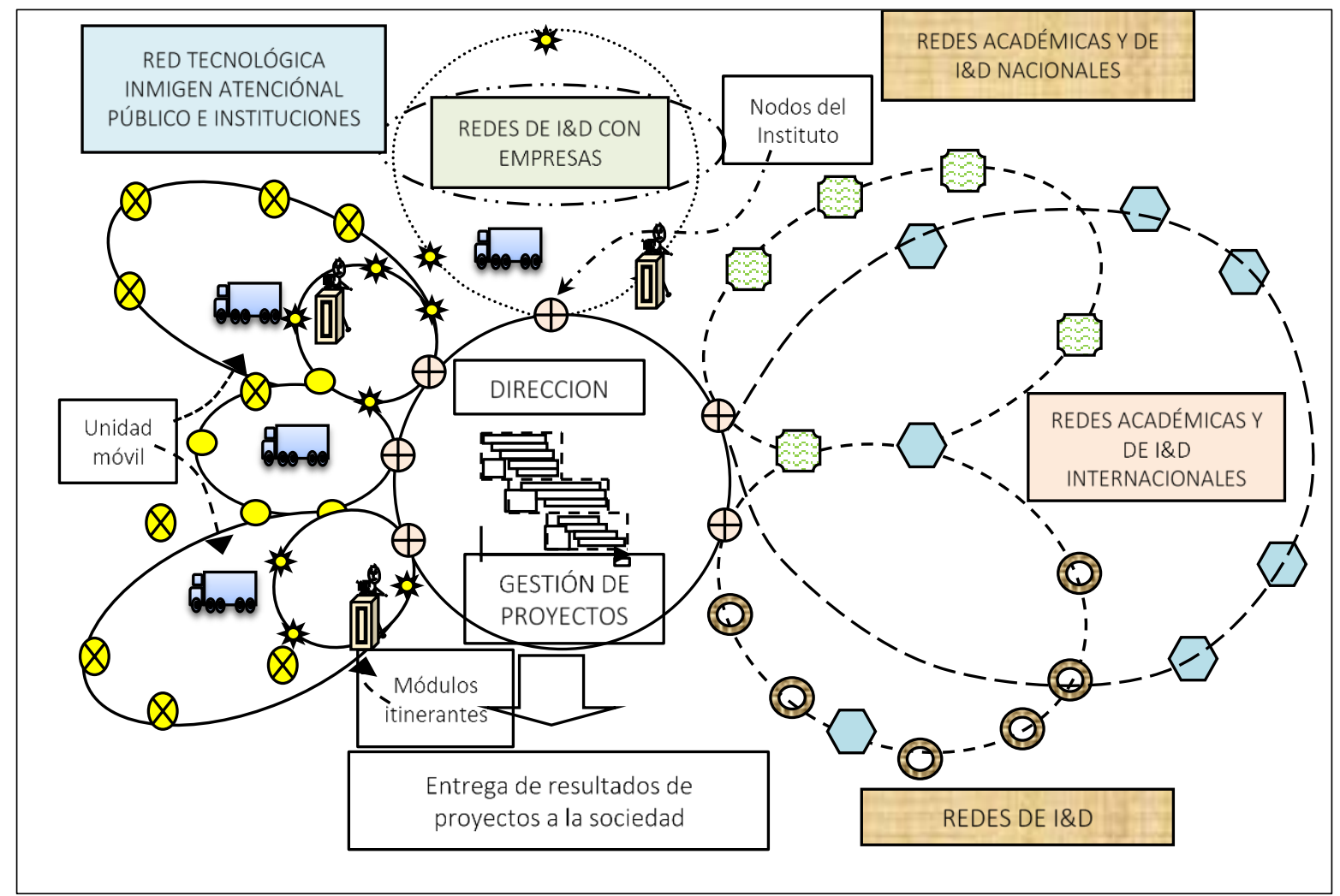

Figura 1. Propuesta de una Red de Vinculación y Gestión de Proyectos para un Instituto de Investigación en salud

Figure 1. Proposal for a linkage and project management network for a health research institute Fuente: elaboración propia. 
En ese sentido, en primer lugar, se deberán establecer, consolidar o incrementar redes de colaboración con instituciones de I\&D públicas o privadas, gubernamentales, que compartan los objetivos de investigación y desarrollo del instituto, y que se encuentren en los diferentes Estados de la República Mexicana; es decir, Redes de I\&D Nacionales. A la vez, deberán hacerse esfuerzos para establecer y desarrollar proyectos de I\&D con empresas y firmas de la iniciativa privada, buscando financiamiento directo o a través de los Fondos Gubernamentales del Consejo Nacional de Ciencia y Tecnología (Conacyt), la Secretaría de Ciencia y Tecnología de la Ciudad de México (Seciti) y Fundaciones, entre otras.

Enseguida se harán esfuerzos por establecer o consolidar redes con instituciones de países latinoamericanos, y en el mediano plazo la institución podrá ser reconocida por su participación en Redes de I\&D Internacionales. El establecimiento de cada red dependerá de proyectos de I\&D estratégicos específicos que sean de alta importancia para la institución. La selección de estos proyectos deberá ser realizada por los cuadros directivos, los grupos académicos y los consejos asesores respectivos.

Dentro de las premisas fundamentales a cumplir en los proyectos que sean desarrollados por las Redes de I\&D, es que más allá de la excelencia en investigación, en el mediano plazo, se deben lograr resultados de conocimiento aplicables y probados con acceso a la sociedad y a los mercados. El problema radica no solo en el desarrollo de las capacidades internas y del acceso a la información y conocimiento de punta, sino en el desarrollo de nuevas formas de gestión de redes para lograr comunicación ágil, coordinación y buen desempeño de los proyectos.

El papel y el perfil de las redes deberán cambiar de acuerdo con las etapas de desarrollo de las innovaciones.

\section{Plan táctico}

Para mejorar el sistema de vinculación y gestión en el plan táctico, se propone llevar a cabo las siguientes acciones específicas:

A. En el corto plazo, de uno a dos años, coordinar, desarrollar y establecer un sistema de cómputo a través del cual se establezca una red de enlaces individuales en los diferentes hospitales, instituciones públicas y empresas de la Ciudad de México y de los diferentes estados de la República Mexicana para llevar a cabo la vinculación horizontal del Instituto en forma puntual. Se propone que el nombre de esta red sea: Red Tecnológica INMEGEN (RTI). Cargar las bases de datos de convenios, los proyectos exitosos, los proyectos en desarrollo, las transferencias tecnológicas, la propiedad intelectual registrada por el instituto y la propiedad intelectual transferida a otros sectores. Bases de datos de académicos científicos y tecnólogos con los que se han realizado y se realizan acciones de I\&D.

B. De mediano a largo plazo, de tres a cinco años, firmar convenios de colaboración con las instituciones que sean parte de la RTI para coordinar las diferentes acciones de colaboración acordadas, como capacitación en línea, así como los diferentes servicios que ofrecerá el INMEGEN. En el convenio se definirán los responsables de la RTI por cada una de las partes y la logística 
empleada para prestar los distintos servicios de laboratorio, asesoría, consultoría, desarrollo tecnológico e investigación.

C. En el corto plazo, de seis meses a un año, coordinar y establecer un sistema de módulos móviles para desarrollar jornadas itinerantes de promoción y pláticas de divulgación de los resultados tecnológicos, productos genómicos y de conocimiento obtenidos en el instituto, a fin de difundir en la población general que acude a las instituciones participantes en la RTI. La Dirección de Vinculación y sus subdirecciones serán las responsables de proponer los planes de movilidad, las acciones a adelantar y el sistema de desempeño y evaluación del quehacer de estos módulos. Los módulos móviles serán manejados por brigadas de dos o tres personas adscritas a la Dirección de Vinculación del Instituto y solo requerirán de un pequeño stand armable y material de difusión y divulgación.

D. En el mediano plazo, de dos a tres años, diseñar, integrar o adquirir, coordinar y establecer un sistema de Unidades Móviles para llevar a cabo jornadas itinerantes de formación de recursos humanos de alta especialidad en medicina genómica, brindando los servicios que en su momento se acuerden.

En el largo plazo, de tres a cinco años, las Unidades Móviles permitirán establecer un primer nivel de contacto y vinculación personal con instituciones del interior de la República que estén asociados y sean parte de la RTI.

E. Se podría desarrollar y definir un Boletín Informativo de Acciones de Vinculación en la que se incluirán "Noticias del INMEGEN», como nuevas tecnologías desarrolladas, patentes, títulos de propiedad intelectual, cursos de formación de recursos humanos impartidos, celebración y firma de convenios, acciones llevadas a cabo por los Módulos y Unidades Móviles, noticias de congresos, información en línea de importancia para el personal del Instituto, premios y distinciones recibidas por los académicos, noticias de la RTI, entre otras.

F. La Dirección de Vinculación será la responsable de realizar la gestión tecnológica de los proyectos que surjan de las acciones de vinculación específicas y puntuales de los Módulos y Unidades Móviles de la Red Tecnológica del INMEGEN propuesta.

\section{DISCUSIÓN}

Para Méndez-Ramírez y Becerril-Sánchez (2005), existe una serie de herramientas gerenciales para que las organizaciones puedan hacer más eficientes sus operaciones, entre otras, la gestión de la calidad total, la reingeniería, la gestión de proyectos, el método Deming, el método Just in Time, el método de Benchmarking, el método Baldrige y la planeación estratégica. Dentro de los anteriores, uno de los procedimientos más utilizados al realizar gestión institucional es la identificación de procedimientos de referencia o benchmark, probados en otras instituciones nacionales o extranjeras; es decir, se identifican acciones llevadas a cabo por otras instituciones de características similares, en términos del tipo de población, nivel de desarrollo económico, objetivos organizacionales, entre otros, y se aplican en la organización bajo estudio.

La planeación estratégica es el proceso mediante el cual los miembros que guían una organización prevén su futuro y desarrollan los métodos y procedimientos para alcanzarlo. Dadas las características de la institución, sus objetivos, su visión, su relativa «juventud» organizacional, su alto nivel científico y su campo específico de aplicación no fue posible encontrar metodologías de gestión 
organizacional para la mejora de la vinculación específicas usadas por instituciones similares; tampoco se pudieron aplicar otras herramientas gerenciales por ser más costosas y en algunos casos de mucho más larga duración. Por lo anterior, en el caso presentado se abordó el problema de modernizar y mejorar la vinculación institucional proponiendo las acciones resultantes de la planeación estratégica para lograr un mayor acercamiento a la población y generar nuevos esquemas de ingresos y la generación y gestión de nuevos proyectos de I\&D.

La planeación estratégica fue el procedimiento metodológico consensuado más adecuado para identificar las acciones requeridas, teniendo en cuenta las restricciones de recursos humanos, económicos y de tiempo. También se identificaron y utilizaron los modelos de gestión de proyectos y de generación de conocimiento presentados en el marco teórico de este trabajo. En el análisis realizado quedó de manifiesto que el modelo de gestión tecnológica de proyectos de la UNAM, y los modelos de innovación abierta basados en la creación de comunidades o redes de innovación son la mejor opción para ser adoptados, asimilados y adaptados en la construcción de las acciones puntuales que se deben adelantar para la ejecución del plan.

Otro aspecto importante, es que, además de favorecer el mayor contacto con la población y el desarrollo de mecanismos de vinculación más eficientes, se espera que la promoción de nuevos proyectos de I\&D deberá impulsar la creación de nuevas tecnologías. Será responsabilidad de Unidades de Vinculación su protección intelectual y su transferencia a otras instituciones y la gestión de licenciamientos para su explotación comercial.

La institución, desde sus orígenes, se enfocó en estudio del cáncer. En consecuencia, a la fecha ha logrado una patente concedida de un método de diagnóstico temprano de carcinoma hepatocelular, cuyos inventores son Pérez Carreón y Sánchez Rodríguez (2013); y dos solicitudes de patente, el método de subclasificación de tumores de mama triple negativos, cuyos inventores son Hidalgo et al., (2016); y el método de pronóstico de cáncer de mama, cuyos inventores son Meléndez Zajgla y Bahena Ocampo (2015).

Dado que los tres problemas de salud principales en México son el cáncer, la obesidad y la diabetes, se espera que en el futuro se generen nuevas tecnologías para atender estas enfermedades y muchas otras, por lo que se deberá promover la creación de capacidades para la transferencia de tecnología. El proceso de transferencia de tecnología interinstitucional es complejo, depende de un número enorme de variables dentro y fuera de la organización, y por lo tanto requiere de la formación de equipos de trabajo interdisciplinarios. Para el Departamento de Asuntos Económicos y Sociales de las Naciones Unidas (Undesa, del inglés) hay cinco temas clave para llevar a cabo acciones de transferencia de tecnología efectivas: (1) desarrollo de recursos humanos, (2) desarrollo institucional, (3) redes de información, (4) identificación y desarrollo de redes de socios e interesados, (5) investigación y desarrollo colaborativas (Undesa, 2008).

Las acciones determinadas en el plan estratégico presentado cumplen con estos cinco requerimientos, por lo que, en otras palabras, más allá de mejorar la vinculación para ofrecer más servicios a la población, se espera que en el mediano y largo plazo se puedan transferir e implantar las tecnologías desarrolladas en el instituto a otras organizaciones para lograr mayor impacto social, asimismo, licenciar tecnologías para su explotación comercial, cumpliendo con los protocolos de ética médica. 


\section{CONCLUSIONES}

Todas las organizaciones requieren de la realización de planes para enfrentarse y adaptarse a momentos de cambio. En este artículo se ha presentado el caso del INMEGEN (2011), cuya situación actual presenta nuevos retos operativos, ya que cambió la presidencia del país, las autoridades del sector salud y la administración del instituto; además, se impuso un programa de austeridad extraordinario. En este contexto, todas las áreas operativas de la institución requieren adaptarse, reformulando sus objetivos y su forma de operar.

Según el enfoque de sistemas dinámicos o cambiantes con el tiempo, la mejor forma de lograr la operación y administración controlada y confiable en sistemas complejos es a través de cambios simples y graduales. La cantidad de variables, usuarios, datos, convenios, investigaciones e interesados tanto internos como externos, hacen que el sistema organizacional del presente caso de estudio sea extraordinariamente complejo. Por esa razón se hizo necesario desarrollar una metodología de planeación simple con acciones que pueden ser replicables, identificando los aspectos clave del problema a resolver, del futuro deseado y las acciones y recursos necesarios para llegar al mismo, a través de etapas de mejora en la vinculación organizacional.

Desde el nacimiento del Instituto y de su Dirección de Vinculación y Desarrollo Interinstitucional, hasta la actualidad, su operación se ha centrado en la firma de diversos instrumentos consensuales para acordar los términos de los proyectos académicos y científicos que se llevan a cabo. El plan propuesto para incentivar, mejorar y sistematizar las acciones de gestión de proyectos y vinculación con la sociedad se basa en dos acciones fundamentales, las cuales respetan las condiciones de austeridad económica y cumplen con la condición de bajo costo de implementación, según ha sido establecido por la administración pública federal.

La primera acción es la construcción de comunidades o redes de innovación con instituciones nacionales e internacionales públicas y privadas, con las cuales existan complementariedades en las capacidades de I\&D o bien sean usuarios potenciales de los servicios que puede prestar el INMEGEN. Estas comunidades o redes permitirán el acceso a fuentes de financiamiento gubernamentales o internacionales e incursionar en campos tecnológicos nuevos. La segunda es llevar a cabo un mayor acercamiento y contacto directo con el público usando unidades móviles y módulos itinerantes, para propiciar el desarrollo de nuevos negocios a través de la oferta de bienes y servicios de base genómica y áreas afines que se generen en el instituto, haciendo énfasis en los diagnósticos y tratamientos dirigidos a la salud de la población, la medicina de precisión y la medicina traslacional. Para la puesta en operación de estas acciones, se plantea que la comunicación debe ser abierta, evolutiva, flexible y multidireccional, y que los gestores requieren desarrollar extraordinarias habilidades de negociación y mediación entre la comunidad interna del instituto y las comunidades de las redes externas y el público en general.

Así, se espera que las implicaciones prácticas de este trabajo para la comunidad académica sean que, por sus diferentes componentes de gestión de proyectos, acciones de vinculación y procedimientos de protección intelectual y transferencia de tecnología, la propuesta de una Red de Vinculación y Gestión de Proyectos realizada para un Instituto de Investigación en salud mexicano pueda ser de utilidad para otros institutos similares o para otras organizaciones. 


\section{REFERENCIAS}

Aguilar Cruz, C. (2000). La necesidad de la planeación estratégica en las organizaciones industriales modernas. $\underline{U R L}$

Belderbos, R.; Carree, M.; Lokshin, B. (2006) Complementarity in R\&D Cooperation Strategies. Review of Industrial Organization, v. 28, 401-426. https://doi.org/10.1007/s11151-006-9102-z

Calvin, S. W.; Wen-Goang, Y.; Kuei-Kuei, L. (2014). Technological position in alliances network. Technology Analysis \& Strategic Management, v. 26, n. 6, 669-685. https://doi.org/10.1080/09537325.2014.923096

Campos Ríos, G.; Sánchez Daza, G. (2005). La Vinculación Universitaria: Ese obscuro objeto del deseo. Revista electrónica de investigación educativa, v. 7, n. 2. URL

Chang, C. K. N.; Shipp, S. S.; Wang, A. J. (2002) The Advanced Technology Program: A public-private partnership for early stage technology development. Venture Capital an International Journal of Entrepreneurial Finance, v. 4, n. 4, 363-370. https://doi.org/10.1080/1369106022000028262

Chesbrough, H. W.; Vanhaverbeke, W.; West, J. (2006) Open innovation: researching a new paradigm. Oxford University Press.

Chesbrough, H. W.; Appleyard, M. M. (2007). Open innovation and strategy. California Management Review, v. 50, n. 1, 57-76. https://doi.org/10.2307/41166416

Chiavenato, I. (2017). Planeación Estratégica, fundamentos y aplicaciones. Tercera Ed. McGraw-Hill.

Das, T. K.; Kumar, R. (2011). Interpartner Negotiations in Alliances: A strategic Framework. Management Decision, v. 49, n. 8, 1235-1256. https://doi.org/10.1108/00251741111163106

De Rond, M.; Bouchikhi, H. (2004). On the dialectics of Strategic Alliances. Organization Science. v. 15, n. 1, 56-69. https://doi.org/10.1287/orsc.1030.0037

Díaz Silva, E.; Doncel Barrera, Y.; Rodríguez, Y. C. (2012). San Juan de Dios: una muestra del problema de Salud en Bogotá y una oportunidad para la educación legal clínica. Revista Universitas estudiantes, n. 9. 47-66. URL

Domínguez Ríos, M. del C., (2013). Prácticas innovadoras en la Vinculación escuelas de negociosempresas. Recherches en Sciences de Gestion-Management Sciences-Ciencias de Gestión, n. 98, 23-42.

Farazi, M. S.; Gopalakrishnan, S.; Perez-Luño, A. (2019). Depth and breadth of knowledge and the governance of technology alliances. Journal of Engineering and Technology Management, v. 54, 28-40. https://doi.org/10.1016/i.jengtecman.2019.08.002 
Fleury, A.; Alaez, C.; Dessein, A.; Rosetti, M.; Saenz, B.; Hernández, M.; Bobes, R. J.; Ramírez-Aquino, R.; Sciutto, E.; Gorodezky, C.; Fragoso, G. (2018). No association of IL2, IL4, IL6, TNF, and IFNG gene polymorphisms was found with Taenia solium human infection or neurocysticercosis severity in a family-based study. Human Immunology, v. 79, n. 7, 578-582.

https://doi.org/10.1016/j.humimm.2018.04.011

Giordani, P. E.; Rullani, F.; Zirulia, L. (2018). Endogenous growth of open collaborative innovation communities: A supply-side perspective. Industrial and Corporate Change, v. 27, n. 4, 745-762. https://doi.org/10.1093/icc/dty004

Hidalgo Miranda, A.; Romero Córdoba, S. L.; Rodríguez Cuevas, S. A.; Maffuz Aziz, A. (2016). Método de subclasificacion de tumores de mama triple negativos. Solicitud de patente MX/a/2016/015339, México: Instituto Mexicano de la Propiedad Industrial. URL

Hofer, C. W.; Schendel, D. (1985). Planeación Estratégica: conceptos analíticos. Editorial Norma.

Huxham, C.; Vangen, S. (2005). Managing to collaborate: The theory and practice of collaborative advantage. Routledge.

Instituto Nacional de Medicina Genómica, INMEGEN (s.f.). El Instituto. URL

INMIGEN (2011). Estatuto Orgánico. URL

Jiménez, S G. (2003). Un paso más hacia el establecimiento del INMEGEN. Informe de Actividades, 2004.

Kaats, E.; Opheij W. (2012). Leren samenwerken tussen organisaties: samen bouwen aan allianties, netwerken, ketens en partnerships. Kluwer.

Kaats, E.; Opheij, W. (2014). Creating Conditions for Promising Collaboration: Alliances, Networks, Chains, and Strategic Partnerships. Springer Briefs in Business.

Kenny, G. (2005). Strategic Planning and Performance Management: Develop and Measure Winning Strategy. Elsevier.

Kim, J. (2015). Performance-based development funding using market mechanisms: A public-private partnership social financing model for medical equipment technology in developing countries. Progress in Development Studies, v. 15, n. 3, 257-269.

https://doi.org/10.1177/1464993415578982

Kim, H.; Choi, S. (2014). Technological alliance portfolio configuration and firm performance. Review of Managerial Science, v. 8, 541-558. https://doi.org/10.1007/s11846-013-0117-1

Lerner, J.; Merges, R. P. (1998). The Control of Technology Alliances: An Empirical Analysis of the Biotechnology Industry. The Journal of Industrial Economics, v. 46, n. 2, 125-156.

https://doi.org/10.1111/1467-6451.00066 
Lichtenthaler, U. (2017). Open innovation: past research, current debates, and future directions. Academy of Management Perspectives. v. 25, n. 1. https://doi.org/10.5465/amp.25.1.75

Lin, H.; Darnall, N. (2014). Strategic Alliance Formation and Structural Configuration. Journal of Business Ethics, v. 127, 549-564. https://doi.org/10.1007/s10551-014-2053-7

Lynn, L. H.; Reddy, N. M.; Aram, J. D. (1996). Linking technology and institutions: The innovation community framework. Research Policy, v. 25, n. 1, 91-106. https://doi.org/10.1016/0048-7333(94)00817-5

MacNaughton, G.; McGill, M. (2019). The Challenge of Interdisciplinarity in Operationalizing the Right to Health. Health and Human Rights, v. 21, n. 2, 251.

Meléndez Zajgla, I. U.; Bahena Ocampo, I. U. (2015). Method for prognosis in breast cáncer. Solicitud de patente MX/E/2015016721. México: Instituto Mexicano de la Propiedad Industrial. URL

Méndez-Ramírez, J. J.; Becerril-Sánchez, T. (2005). Planeación estratégica: técnica gerencial aplicada en la administración pública. Quivera Revista de Estudios Territoriales, v. 7, n. 2, 160-178. URL

Mintzberg, H. (1994). Rethinking strategic planning part II: New roles for planners. Long Range Planning, v. 27, n. 3, 22-30. https://doi.org/10.1016/0024-6301(94)90186-4

Morcillo Ortega, P. (1997). Dirección Estratégica de la tecnología e Innovación: un enfoque de competencias. Civitas Ediciones.

Nelson, J. C.; Raskind-Hood, C.; Galvin, V. G.; Essien, J. D.; Levine, L. M. (1999). Positioning for Partnerships: Assessing Public Health Agency Readiness. American Journal of Preventive Medicine, v. 16, n. 3, 103-117. https://doi.org/10.1016/s0749-3797(99)00003-3

Pérez Carreón, J. I.; Sánchez Rodríguez, R. (2013). Método de diagnóstico temprano de carcinoma hepatocelular. MX 357018 B, México: Instituto Mexicano de la Propiedad Industrial.

Petraite, M., (2013). R\&D innovation through networks and collaboration: linking innovation capabilities globally. XXIV ISPIM Conference - Innovating in Global Markets: Challenges for Sustainable Growth in Helsinki, Finland on 16-19 June 2013. Available to ISPIM members at www.ispim.org.

Rodríguez Pérez, M. A.; Acuña Martínez, J. A.; Rojas Vera, L. R.; Lobato, L. A. (2015). Vinculación Universidad - Empresa - Estado, en Nicaragua. Orbis Revista Científica Electrónica de Ciencias Humanas, n. 31, 15-34. URL

Rojas, A. G. (2018). Toma de protesta de AMLO: Andrés Manuel López Obrador saca su parte más indigenista en la fiesta del Zócalo. $\underline{\text { URL }}$

Sabater Quinto, F.; Monserrat Gauchi, J. (2017). Planificación estratégica de la comunicación en redes sociales. Editorial UOC. 
Sanders, T. I. (1998). Strategic Thinking and the New Science: Planning in the Midst of Chaos Complexity and Change. The Free Press.

Séguin, B.; Hardy, B. J.; Singer, P. A.; Daar, A. S. (2008). Genomics, public health and developing countries: the case of the Mexican National Institute of Genomic Medicine (INMEGEN). Nature Reviews Genetics, v. 9, n. S5-S9. https://doi.org/10.1038/nrg2442

Staines Boone, A.T.; Chinn, I. K.; Alaez-Versón, C.; Yamazaki-Nakashimada, M. A.; Carrillo-Sánchez, K.; García-Cruz, M. L. H.; Poli, M. C.; González Serrano, M. E.; Medina Torres, E. A.; Muzquiz Zermeño, D.; Forbes, L. R.; Espinosa-Rosales, F. J.; Espinosa-Padilla, S. E.; Orange, J. S.; Lugo Reyes, S. O. (2019). Failing to Make Ends Meet: The Broad Clinical Spectrum of DNA Ligase IV Deficiency. Case Series and Review of the Literature. Frontiers in Pediatrics. v. 6.

https://doi.org/10.3389/fped.2018.00426

Stern, C. W.; Stalk, Jr. G. (1998). Ideas sobre estrategia: The Boston Consulting Group. Ediciones Deusto.

Suárez-Balcázar, Y.; Harper, G. W.; Lewis, R. (2005). An Interactive and Contextual Model of Community-University Collaborations for Research and Action. Health Education Behavior, v. 32, n. 1, 84-101. https://doi.org/10.1177/1090198104269512

Taylor, B. (1991). Planeación Estratégica Exitosa. Fondo Editorial Legis.

Taylor-Alexander, S.; Schwartz-Marín, E. (2013). Bioprophecy and the politics of the present: notes on the establishment of Mexico's national genomics institute (INMEGEN). New Genetics and Society, v. 32, n. 4, 333-349. https://doi.org/10.1080/14636778.2013.845076

United Nations Department of Economic and Social Affairs. (2008). Climate Change: Technology Development and Technology Transfer. United Nations Department of Economic and Social Affairs. $\underline{U R L}$

Valenzuela-Ponce, H.; Alva-Hernánde, S.; Garrido-Rodríguez, D.; Soto-Nava, M.; García-Téllez, T.; Escamilla-Gómez, T.; García-Morales, C.; Quiroz-Morales, V. S.; Tapia-Trejo, D.; del ArenalSánchez, S.; Prado-Galbarro, F. J.; Hernández-Juan, R.; Rodríguez-Aguirre, E.; MurakamiOgasawara, A.; Mejía-Villatoro, C.; Escobar-Urias, I. Y.; Pinzón-Meza, R.; Pascale, J. M.; Zaldivar, Y.; Porras-Cortés, G.; Quant-Durán, C.; Lorenzana, I.; Meza, R. I.; Palou, E. Y.; Manzanero, M.; Cedillos, R. A.; Aláez, C.; Brockman, M. A.; Harrigan, P. R.; Brumme, C. J.; Brumme, Z. L.; Ávila-Ríos, S.; ReyesTerán, G. (2018). Novel HLA class I associations with HIV-1 control in a unique genetically admixed population. Scientific Reports, v. 8. https://doi.org/10.1038/s41598-018-23849-7 
Velázquez, V. G. (2012). Impacto de la comunicación organizacional, en la competitividad y la vinculación de las empresas mexicanas con la universidad. Recherches en Sciences de Gestion, n. 90, 89-119. https://doi.org/10.3917/resg.090.0087

Villegas, P. (2018). La toma de posesión de AMLO: combate a la corrupción, pero no perseguirá a los antiguos funcionarios. $\underline{U R L}$

Wohlstetter, P.; Smith, J.; Malloy, C. L. (2005). Strategic Alliances in Action: Toward a Theory of Evolution. The Policy Studies Journal, v. 33, n. 3, 419-442. https://doi.org/10.1111/j.1541-0072.2005.00123.x

Wu, B.; Gong, C. (2019). Impact of Open Innovation Communities on Enterprise Innovation Performance: A System Dynamics Perspective. Sustainability, v. 11, n. 17, 4794. https://doi.org/10.3390/su11174794

Yusuf, S. (2008). Intermediating Knowledge Exchange between Universities and Businesses. Research Policy, v. 37, n. 8, 1167-1174. https://doi.org/10.1016/j.respol.2008.04.011 